International Journal of Diabetology \& Vascular Disease Research (IJDVR)

ISSN:2328-353X

\title{
Rhabdomyolysis as an Unusual Complication of Hyperosmolar Hyperglycemic State
}

Ahmed Abdel-Fattah Denewar, Nagib AM*, Aliaa Risk Ismael, Abbas MH, Refaie AF

Department of Dialysis and Transplantation, The Urology - Nephrology Center, Mansoura University, Egypt.

\section{Abstract}

Objectives: Hyperosmolar hyperglycemic state (HHS) is one of the serious metabolic derangements that occurs in patients with diabetes mellitus, Cases associated with hyperosmolarity due to diabetic ketoacidosis or hyperosmolar hyperglycemic state have also been described, and hypophosphatemia may contribute to the risk of rhabdomyolysis in some of these patients, Rhabdomyolysis is a potentially life-threatening syndrome characterized by muscle necrosis and the release of intracellular muscle contents into the circulation, associated acute kidney injury (AKI) and severe electrolyte derangements are life-threatening, and are accompanied by extreme elevations in CK levels.

Case Report: A 47 year-old Egyptian gentleman, admitted to our center ICU with obtundation and dehydration with a 4-day history of generalized weakness, nausea and persistent vomiting. The patient had strong family history of type 2 diabetes mellitus. On admission, Neurological evaluation was irrelevant. Radiological evaluations revealed left basal pneumonic patch. His relatives deny any history of drug or alcohol abuse. Laboratory evaluation fulfilled the proposed diagnostic criteria for acute kidney injury, rhabdomyolysis and Hyperosmolar hyperglycemic state.

Conclusion: Type 2 diabetes mellitus may be presented for the first time by serious complication as HHS which in turn can cause a sever morbidities like rhabdomyolysis and acute kidney injury.

Keywords: Hyperglycemic State; Rhabdomyolysis; Acute Kidney Injury.

\section{*Corresponding Author:}

Ayman Maher Nagib MD,

Consultant Nephrologist, Urology Nephrology Center, Mansoura, Egypt Tel: +20502262222

Fax: +2050223717

E-mail: ayman_maher2005@yahoo.com

Received: October 15, 2015

Accepted: November 03, 2015

Published: November 03, 2015

Citation: Nagib AM, et al., (2015) Rhabdomyolysis as an Unusual Complication of Hyperosmolar Hyperglycemic State. Int J Diabetol Vasc Dis Res, 3(9) 127-129. doi: http:/ / dx.doi.org/10.19070/2328-353X-1500027

Copyright: Nagib $\mathbf{A M}^{\odot}$ 2015. This is an open-access article distributed under the terms of the Creative Commons Attribution License, which permits unrestricted use, distribution and reproduction in any medium, provided the original author and source are credited.

\section{Introduction}

Hyperosmolar hyperglycemic state (HHS) is one of the serious metabolic derangements that occurs in patients with diabetes mellitus (DM) and can be a life-threatening emergency, it is less common than the other acute complication of diabetes, diabetic ketoacidosis (DKA), HHS was previously termed hyperosmolar hyperglycemic nonketotic coma (HHNC); however, the terminology was changed because coma is found in fewer than $20 \%$ of patients with HHS [1]. Guidelines on the management of Hyperglycemic Hyperosmolar State (HHS) in adults are uncommon and often there is little to differentiate them from the management of DKA. However, HHS is different and treatment requires a differ- ent approach. Although typically occurring in the elderly, HHS is presenting in ever younger adults and teenagers often as the initial presentation of Type 2 diabetes mellitus (T2DM) [2, 3]. Rhabdomyolysis has been associated with a variety of electrolyte disorders, particularly hypokalemia and hypophosphatemia [4]. Cases associated with hyperosmolarity due to diabetic ketoacidosis or hyperosmolar hyperglycemic state have also been described, and hypophosphatemia may contribute to the risk of rhabdomyolysis in some of these patients [5]. Acute kidney injury (AKI) and severe electrolyte derangements are life threatening, and are accompanied by extreme elevations in CK levels [6]. We report a rare case of HHS as initial presentation of Type 2 diabetes mellitus complicated with rhabdomyolysis and acute kidney injury.

\section{Case Presentation}

A 47 year-old Egyptian gentleman, admitted to Mansoura urology and nephrology center ICU with obtundation and dehydration with a 4-day history of generalized weakness, nausea and persistent vomiting. The patient had irrelevant medical and surgical history with strong family history of Type 2 diabetes mellitus. On admission, his blood pressure was 115/68 $\mathrm{mmHg}$, his pulse rate was 109 beat/min that was regular with bilateral equality; his respiratory rate was 44 breathes/min with rapid deep pattern; his temperature was 35.8 centigrade degree. Chest auscultation showed bilateral vesicular breathing with few scattered sibilant rhonchi increased in intensity towards the center of the chest. Abdominal examination revealed no abnormalities. Neurological evaluation revealed no signs of lateralization, no signs of meningeal irritation with GCS 14 (E4M6V4). His BMI was $26.1 \mathrm{~kg} / \mathrm{m}^{2}$. ECG was free. Oropharyngeal examination showed extremely dry mucus 
membranes with mild oral moniliasis. Lower limbs were dry with no swellings or signs of trauma. Axillae were dry. Radiological evaluations including abdominal sonography and CT brain were free. Non contrast CT chest revealed left basal pneumonic patch. His relatives deny any history of drug or alcohol abuse. Laboratory evaluation fulfilled the proposed diagnostic criteria for acute kidney injury, rhabdomyolysis and HHS Table [1].

A case of HHS, acute kidney injury, hypernatremia and rhabdomyolysis was diagnosed. A fluid resuscitation with half normal saline (saline $0.45 \%$ ) was commenced with regular insulin infusion at a rate of 7 units $/$ hour $(0.1 \mathrm{U} / \mathrm{kg})$ after 7 units of bolus regular insulin was started. Empiric antibiotic coverage of community acquired pneumonia was ordered. Sodium bicarbonate for urine alkalinization was avoided because of life-threatening hypernatremia with unavailability of sodium free bicarb.

\section{Preparation}

On the $2^{\text {nd }}$ day, fortunately, kidney functions started to improve (Scr $2.3 \mathrm{mg} / \mathrm{dl}$ ), uric acid $13.4 \mathrm{mg} / \mathrm{dl}$, serum $\mathrm{Na}^{+}: 158 \mathrm{MEq} / \mathrm{L}$, blood glucose: $379 \mathrm{mg} / \mathrm{dl}$, urine output was $3150 \mathrm{cc} /$ day and CPK level decreased to $10699 \mathrm{IU} / \mathrm{L}$.

After 11 days of treatment, serum creatinine showed maximum decrease to reach $0.5 \mathrm{mg} / \mathrm{dl}$, serum $\mathrm{Na}^{+}: 142 \mathrm{MEq} / \mathrm{L}$, uric acid $4.4 \mathrm{mg} / \mathrm{dl}$, blood glucose: $179 \mathrm{mg} / \mathrm{dl}, \mathrm{CPK}: 363 \mathrm{IU} / \mathrm{L}$ and the patient regained his full consciousness level after which he was referred home.

\section{Discussion}

Our patient presented on admission with acute kidney injury and rhabdomyolysis due to first discovered Type 2 diabetes mellitus with HHS as initial presentation. Hyperosmolar hyperglycemic state (also called non-ketotic hyperglycemia) is one of the most serious acute complications of diabetes. In HHS, there is little or no ketoacid accumulation, the serum glucose concentration frequently exceeds $1000 \mathrm{mg} / \mathrm{dL}$ (56 mmol/L), the plasma osmolality may reach $380 \mathrm{mosmol} / \mathrm{kg}$, and neurologic abnormalities are frequently present (including coma in 25 to 50 percent of cases) [7]. Our case presented by changes in mental performance as neurologic deterioration primarily occurs in patients with an effective plasma osmolality above 320 to $330 \mathrm{mosmol} / \mathrm{kg}$ [8]. Mental obtundation and coma are more frequent in HHS than DKA because of the usually greater degree of hyperosmolality in HHS [9].

Our patient presented by left basal pneumonic patch which can be considered as the precipitated factor as HHS is usually precipitated by stresses that act in part by increasing the secretion of glucagon, catecholamines, and cortisol. Infection, such as pneumonia, gastroenteritis, and urinary tract infection, can be found in 40 to 50 percent of patients with hyperglycemic crisis; other stresses include pancreatitis, myocardial infarction, stroke, trauma, and alcohol and drug abuse [10].

Nonexertional and nontraumatic causes of rhabdomyolysis include drugs and toxins, infections, electrolyte abnormalities, endocrinopathies, inflammatory myopathies, and others [11]. Rhabdomyolysis has been associated with a variety of electrolyte

Table 1. Laboratory findings on admission and follows up.

\begin{tabular}{|c|c|c|c|}
\hline & $\mathbf{0}$ day & $\mathbf{2}^{\text {nd }}$ day & $\mathbf{1 1}^{\text {th }}$ days \\
\hline Serum. creatinine & $4.2 \mathrm{mg} / \mathrm{dl}$ & $2.3 \mathrm{mg} / \mathrm{dl}$ & $0.5 \mathrm{mg} / \mathrm{dl}$ \\
\hline Serum. sodium & $164 \mathrm{MEq} / \mathrm{L}$ & $158 \mathrm{MEq} / \mathrm{L}$ & $142 \mathrm{MEq} / \mathrm{L}$ \\
\hline Serum. potassium & $3.3 \mathrm{MEq} / \mathrm{L}$ & & \\
\hline Blood sugar & $1127 \mathrm{mg} / \mathrm{dl}$. & $379 \mathrm{mg} / \mathrm{dl}$ & $179 \mathrm{mg} / \mathrm{dl}$ \\
\hline Uric acid & $16.9 \mathrm{mg} / \mathrm{dl}$. & $13.4 \mathrm{mg} / \mathrm{dl}$ & $4.4 \mathrm{mg} / \mathrm{dl}$ \\
\hline Corrected total calcium & $9.4 \mathrm{mg} / \mathrm{dl}$. & & \\
\hline Phosphorus & $3 \mathrm{mg} / \mathrm{dl}$. & & \\
\hline BUN & $151 \mathrm{mg} / \mathrm{dl}$. & & \\
\hline Chloride & $107 \mathrm{mg} / \mathrm{dl}$. & & \\
\hline Calc. osmolality & $471 \mathrm{Mosm} \backslash \mathrm{L}$ & & \\
\hline Acetone in urine & Negative. & & \\
\hline PH & 7.45 & & \\
\hline HCO & $27.7 \mathrm{Mmol} / \mathrm{L}$ & & \\
\hline PCo & $39.2 \mathrm{MmHg}$ & & \\
\hline CPK & $17000 \mathrm{U} \backslash \mathrm{L}$ & $10699 \mathrm{IU} / \mathrm{L}$ & $\mathrm{CPK:} 363 \mathrm{IU} / \mathrm{L}$ \\
\hline LDH & $521 \mathrm{U} \backslash \mathrm{L}$. & & \\
\hline Hemoglobin & $10.7 \mathrm{gm} \%$ & & \\
\hline MSU dipstick & Hemoglobin casts & & \\
\hline MSU microscopic ex, & $\mathrm{RBCs:} 1-2 / \mathrm{HPF}$. & & \\
\hline Urinary myoglobin & Positive. & & \\
\hline
\end{tabular}

0 day: on admission, CPK: Creatine phosphokinase. S.Cr: serum creatinine. LDH: Lactate dehydrogenase BUN: blood urea nitrogen. PCo2: partial pressure of carbon dioxide. MSU: Midstream Specimen of Urine 
disorders, particularly hypokalemia and hypophosphatemia [4]. Cases associated with hyperosmolarity due to diabetic ketoacidosis or hyperosmolar hyperglycemic state have also been described, and hypophosphatemia may contribute to the risk of rhabdomyolysis in some of these patients [5]. Potassium release from muscle cells during exercise normally mediates vasodilation and the appropriately increased blood flow to muscles. Decreased potassium release due to profound hypokalemia (serum potassium less than $2.5 \mathrm{mEq} / \mathrm{L}$ ) may promote the development of rhabdomyolysis by decreasing blood flow to muscles in response to exertion, in both hypokalemic and hypophosphatemia rhabdomyolysis, the serum potassium and phosphate levels may underestimate or mask the underlying total body depletion because of the release of these electrolytes from intracellular stores due to the myonecrosis [4]. Other electrolyte disorders have been occasionally associated with rhabdomyolysis. These include hypocalcaemia; hyponatremia mostly due to primary polydipsia [12], and hypernatremia [13].

Until now, AKI secondary to HHS-induced rhabdomyolysis has rarely been reported. Although the exact mechanisms by which rhabdomyolysis impairs glomerular filtration rate (GFR) are unclear, some evidence suggests that the mechanisms of renal damage may include: 1) intrarenal vasoconstriction and ischemia; 2) direct and indirect ischemic tubule injury; and 3) tubular obstruction [14].

\section{Conclusion}

Type 2 diabetes mellitus may be presented for the first time by serious complication as HHS which in turn can cause a sever morbidities like rhabdomyolysis and acute kidney injury.

\section{References}

[1]. Nugent BW (2005) Hyperosmolar hyperglycemic state. Emerg Med Clin North Am 23(3): 629-648.

[2]. Ekpebergh CO, Longo-Mbenza B, Akinrinmade A, Blanco-Blanco E, Badri M, et al. (2010) Hyperglycaemic crisis in the Eastern Cape province of South Africa: High mortality and association of hyperosmolar ketoacidosis with a new diagnosis of diabetes. S Afr Med J 100(12): 822-826.

[3]. Zeitler P, Haqq A, Rosenbloom A, Glaser N (2011) Hyperglycemic Hyperosmolar Syndrome in Children: Pathophysiological considerations and guidelines for treatment. J Pediatr 158(1): 9-14.

[4]. Singhal PC, Kumar A, Desroches L, Gibbons N, Mattana J (1992) Prevalence and predictors of rhabdomyolysis in patients with hypophosphatemia. Am J Med 92(5): 458-464.

[5]. Casteels K, Beckers D, Wouters C, Van Geet C (2003) Rhabdomyolysis in diabetic ketoacidosis. Pediatr Diabetes 4(1): 29-31.

[6]. Bosch X, Poch E, Grau JM (2009) Rhabdomyolysis and acute kidney injury. N Engl J Med 361: 62-72.

[7]. Kitabchi AE, Young R, Sacks H, Morris L (1979) Diabetic ketoacidosis: reappraisal of therapeutic approach. Ann Rev Med 30: 339-357.

[8]. Kitabchi AE, Razavi NL (2000) Hyperglycemic Crises: Diabetic Ketoacidosis (DKA), and Hyperglycemic Hyperosmolar State (HHS). In:file://www. endotext.org/diabetes/diabetes24/diabetesframe24.htm (Accessed on January 30,2013$)$.

[9]. Lorber D (1995) Nonketotic hypertonicity in diabetes mellitus. Med Clin North Am 79(1): 39-52.

[10]. Nyenwe EA, Loganathan RS, Blum S, Ezuteh DO, Erani DM, et al. (2007) Active use of cocaine: an independent risk factor for recurrent diabetic ketoacidosis in a city hospital. Endocr Pract 13(1): 22-29.

[11]. Khan FY (2009) Rhabdomyolysis: a review of the literature. Neth J Med 67(9): 272-283.

[12]. Putterman C, Levy L, Rubinger D (1993) Transient exercise-induced water intoxication and rhabdomyolysis. Am J Kidney Dis 21(2): 206-209.

[13]. Denman JP (2007) Hypernatraemia and rhabdomyolysis. Med J Aust 187(9): 527-528.

[14]. Ardalan MR, Ghabili K, Mirnour R, Shoja MM (2011) Hypothyroidism induced rhabdomyolysis and renal failure. Ren Fail 33(5): 553-554. 